\title{
Confiabilidade intra e interexaminador da algometria para mensurar o limiar da dor em idosos institucionalizados
}

\author{
Intra and inter-rater reliability of \\ algometry to measure pain threshold in \\ institutionalized elderly
}

\section{Yandra Alves Prestes ${ }^{1}$ \\ Marilene dos Santos Souza ${ }^{2}$ (1) \\ Mayara Souza Pereira ${ }^{3}$ (1) \\ Rosiane Ervati ${ }^{4}$ (1) \\ Hércules Lázaro Morais Campos 5 (1)}

\begin{abstract}
${ }^{1}$ Autora para correspondência. Universidade Federal do Amazonas. Coari, Amazonas, Brasil. yprestess18@hotmail.com 2,5Universidade Federal do Amazonas (Coari). Amazonas, Brasil. marilene.ss1511@gmail.com, herculeslmc@hotmail.com 3,4Universidade Federal do Espírito Santo (Vitória). Espírito Santo, Brasil. mayara.fisio@hotmail.com, rosi_ervati@hotmail.com
\end{abstract}

RESUMO | INTRODUÇÃO: A dor passa ser um fator importante quando se estuda a população idosa, pois apresenta um grande impacto nas atividades diárias, aumentando as comorbidades e a mortalidade, transformando-se em um sério problema de saúde pública. OBJETIVOS: Avaliar a confiabilidade das medidas intraexaminador e interexaminador de um algômetro digital em mensurar o limiar da dor no quadril de idosos institucionalizados e frágeis. MATERIAIS E MÉTODOS: A amostra foi de forma intencional de acordo com a capacidade cognitiva dos idosos para responderem às avaliações; dois examinadores realizaram a algometria na região do quadril em 17 idosos residentes de uma instituição de longa permanência. RESULTADOS: Os coeficientes de correlação intraclasse (CCI) demonstraram alta confiabilidade do instrumento intra-examinador - iguais a 0,877 e muito alta interexaminador - iguais a 0,973. CONCLUSÃO: Há confiabilidade no algômetro digital em mensurar o limiar de dor no quadril em idosos de uma instituição de longa permanência.

PALAVRAS-CHAVE: Confiabilidade. Algometria. Dor. Idosos.

\begin{abstract}
INTRODUCTION: Pain becomes an important factor when studying the elderly population, as it has a great impact on daily activities, increasing comorbidities and mortality, becoming a serious public health problem. OBJECTIVES: To evaluate the reliability of the intra-examiner and inter-examiner measurements of a digital algometer in measuring the hip pain threshold of institutionalized and fragile elderly people. MATERIALS AND METHODS: The sample was intentionally in accordance with the elderly's cognitive ability to respond to assessments; two examiners performed algometry in the hip region on 17 elderly residents of a long-term institution. RESULTS: The intraclass correlation coefficients (ICC) demonstrated high reliability of the intraexaminer instrument - equal to 0.877 and very high interexaminer - equal to 0.973 . CONCLUSION: There is reliability in the digital algometer in measuring the hip pain threshold in the elderly in a long-term institution.
\end{abstract}

KEYWORDS: Reliability. Algometry. Pain. Aged. 


\section{Introdução}

A população brasileira está em um processo acelerado de envelhecimento relacionado, inteiramente, a três fatores como: a redução na taxa de fecundidade, que passou de 6,28 filhos por mulher em 1960 para 1,90 filhos em 2010; aumento da expectativa de vida que chegou a 73,4 anos e a redução na taxa de mortalidade ${ }^{1}$. Atualmente, os dados da Organização Mundial de Saúde - OMS (2018) sugerem que a proporção da população mundial com mais de 60 anos dobrará de 12\% para 22\%, entre 2015 e 2050². De acordo com o Instituto Brasileiro de Geografia e Estatística (IBGE), até 2060, o percentual de pessoas com mais de 65 anos passará dos atuais 9,2\% para 25,5\%. Ou seja, 1 em cada 4 brasileiros será idoso³. Logo, vem crescendo a demanda por instituições para o atendimento e acompanhamento integral do idoso, com o compromisso de suprir suas necessidades básicas e a necessidade de se conhecer melhor essa população ${ }^{4}$.

As instituições asilares têm o dever de garantir o bemestar do idoso com o intuito de promover sua dignidade e inserção social. Elas devem, também, levar em consideração as características próprias do envelhecimento, garantindo um envelhecimento digno ${ }^{5}$.

A dor passa ser um fator importante, quando se estuda a população idosa, pois apresenta um grande impacto nas atividades diárias, aumentando as comorbidades e a mortalidade, transformando-se em um sério problema de saúde pública que precisa ser diagnosticado, mensurado, avaliado e tratado com o intuito de reduzir a morbidade e melhorar a qualidade de vida ${ }^{6}$. Estudos mostram que $50 \%$ dos idosos relatam a dor como queixa principal ${ }^{7}$. Esse é um dos fatores que mais interfere em sua qualidade de vida e suas repercussões têm sido associadas à depressão, à incapacidade e à mortalidade ${ }^{8}$.

A dor é considerada uma experiência multidimensional, sendo descrita em termos de lesões teciduais, reais ou potenciais. A dor é subjetiva, cada indivíduo aprende a expressar e lidar com sua dor a partir de suas experiências anteriores. A dor crônica é definida como aquela que persiste além do tempo razoável para a cura de uma lesão?
A dor decorrente de uma fratura do fêmur proximal é o tipo mais comum em pessoas com mais idade ${ }^{10}$, aumentando com o passar dos anos. Isso pode ser explicado devido à maior prevalência de osteoporose e ao aumento do número de quedas associadas ao problema ${ }^{11}$, acarretando na dor no pós-queda ${ }^{12}$.

A experiência de dor é comum em pessoas com 65 anos ou mais. Em torno de $85 \%$ das pessoas nesta faixa etária terão algum problema de saúde associado a um quadro de dor. A dor acomete entre 32\% a $34 \%$ dos idosos e é caracterizada como ocasional em $20 \%$ a $25 \%$; aguda em $6 \%$ a $7 \%$ e crônica entre $48 \%$ a $55 \%$ dos idosos $\frac{13}{}$.

Discute-se muito que a avaliação da dor é subjetiva, mas falta um instrumento que possa, de fato, quantificar a dor que o idoso relata, por isso, surge a necessidade de validar um instrumento que possa quantificar a dor entre os velhos. A mensuração dessa dor pode influenciar as decisões de cuidado em saúde para essa população ${ }^{\frac{14}{}}$.

Pretende-se avaliar a confiabilidade das medidas intraexaminador e interexaminador do algômetro da marca Instrutherm DD-200® em quantificar a dor de idosos residentes em uma instituição de longa permanência.

\section{Métodos}

Trata-se de um estudo do tipo observacional exploratório, aprovado pelo Comitê de Ética em Pesquisa da Universidade da Cidade de São Paulo (UNICID) registro de número: 096365/2015 (CAAE 49467215.2.0000.0064). A coleta foi realizada durante os meses de julho e agosto de 2015.

Para a caracterização sociodemográfica dos idosos, foi aplicado um questionário semiestruturado para obter as seguintes informações: gênero, faixa etária, grau de instrução, naturalidade, renda mensal, situação de moradia e doenças autorrelatadas.

A pesquisa foi conduzida em uma instituição de longa permanência na cidade da grande Vitória, onde os 
responsáveis pela mesma assinaram o termo de consentimento livre e esclarecido (TCLE). Participaram do estudo 17 idosos que eram capazes de responder, ativamente, à avaliação inicial e de compreender como se processariam as medidas da algometria.

A amostra se deu de forma intencional e de acordo com a capacidade intelectual e a disponibilidade dos idosos em participar do estudo.

Dois examinadores, estudantes do curso de graduação de Fisioterapia da UFES, realizaram um treinamento prévio da algometria por um período de, aproximadamente, uma semana.

Nesse estudo, os idosos necessitavam do cognitivo preservado para que pudessem participar, ativamente, da avaliação. O desempenho cognitivo foi avaliado por meio do Mini exame do Estado Mental (MEEM): é um questionário de rastreamento e de avaliação da função cognitiva, composto por domínios de: orientação espacial; orientação temporal; memória imediata e evocação; cálculo; linguagem-nomeação; repetição, compreensão, escrita e cópia de desenho. Os pontos de cortes são 20 pontos para analfabetos; 21 pontos para indivíduos com um a três anos de escolaridade; 24 pontos para quatro a sete anos de escolaridade e 26 pontos para oito anos de escolaridade ou mais ${ }^{15}$.

Aplicou-se, ainda, a Escala de Depressão Geriátrica (GDS): é um instrumento utilizado para detectar diagnóstico de depressão em indivíduos adultos e idosos. É composto por 15 perguntas negativas/ afirmativas em que o resultado totaliza 5 ou mais pontos, em que um score igual ou maior que $5 \mathrm{ca}$ racteriza depressão grave ${ }^{16}$.

Usou-se o Índice de Comorbidade Funcional (ICF): é um questionário que contempla aspectos multidimensionais da condição de saúde do idoso, sendo constituído por 20 questões distribuídas em oito seções. Ao final, calcula-se, também, o Índice de Massa Corporal (IMC). Os pontos de cortes são de 1 ponto para cada doença autorreferida sobre a presença ou não dessa comorbidade, totalizando 18 pontos, sendo que, quanto maior o número de pontos, mais alto é o índice de vulnerabilidade de comorbidade funcional do indivíduo ${ }^{17}$.
Avaliou-se o desempenho físico-funcional através do Short Physical Performace Battery (SPPB): é um instrumento com testes que avaliam o desempenho físico-funcional por meio do tempo de equilíbrio, da velocidade da marcha e da força nos membros inferiores. Primeiramente, solicita-se ao indivíduo que fique com os pés juntos olhando para frente, se for capaz de se manter na posição durante 10 segundos, será atribuído 1 ponto; caso contrário ou se recusar-se a fazer, nenhum ponto será atribuído. No segundo teste, o indivíduo deve permanecer em posição Semi Tandem, que tem pontuação igual à anterior; solicitaIhe que fique na posição Tandem, um pé à frente do outro; se for capaz de se manter na posição por 10 segundos, serão atribuídos 2 pontos; se mantiver-se na posição, entre 3 a 9,99 segundos, será atribuído 1 ponto e, para um tempo menor que 3 segundos, nenhum ponto será atribuído. Dessa forma, o valor total do teste de equilíbrio será dado pela somatória entre as três posições, apresentando como pontuação máxima 4 pontos. Já a velocidade da marcha é observada ao solicitar que o indivíduo ande, com o seu passo habitual, uma distância de 4 metros. Serão cronometrados os tempos das caminhadas de ida e volta, será válido o menor tempo de caminhada. A pontuação máxima será de 4 pontos, em que serão atribuídos pontos da seguinte maneira: tempo de caminhada menor que 4,82 segundos - 4 pontos; entre 4,82 e 6,20 segundos - 3 pontos; entre 6,21 e 8,70 - 2 pontos; maior que 8,70 - 1 ponto. Caso o indivíduo avaliado não realize a caminhada, nenhum ponto será atribuído. Já o teste de força dos membros inferiores é realizado por meio do sentar-se e do levantar-se da cadeira cinco vezes consecutivas sem utilizar os membros superiores; a pontuação máxima será de 4 pontos atribuídos para um tempo de teste de 11,19 segundos ou menos; 3 pontos atribuídos para um tempo de teste de 11,20 a 13,69 segundos; 2 pontos para um tempo de teste de 13,70 a 16,69 segundos e 1 ponto para 16,70 segundos ou mais. A pontuação total final do SPPB varia de 0 a 11; quanto menor for a pontuação, maior o comprometimento físico-funcional ${ }^{18}$.

Realizou-se a algometria no quadril19. Ela foi utilizada para avaliar o limiar de dor (hiperalgesia) em seis pontos localizados a $2-3 \mathrm{~cm}$ da incisão cirúrgica em três níveis (superior, meio e inferior), utilizando-se 
um algômetro portátil (dinamômetro digital reversível DD500, marca Instrutherm ${ }^{\circledR}$ ) com uma sonda de área de $0,28 \mathrm{~cm} 2$. O limiar de dor foi identificado a partir da aplicação de uma pressão em cada região de forma progressiva e foi interrompida quando o idoso manifestou perceber o estímulo de dor. Os participantes foram orientados a verbalizarem quando apresentassem a primeira sensação desagradável de dor (percepção dolorosa). O avaliador utilizou um ângulo de aproximação de 90 graus, formado entre a superfície de estimulação e o ponto estimulado, com velocidade constante. O idoso foi avaliado na posição mais confortável possível, sentado ou deitado. Quando na posição deitada (a maioria dos idosos), o participante permaneceu em decúbito lateral sobre o hemicorpo contralateral à cirurgia como se pode ver na figura 1.

Figura 1. Seis pontos demarcados sobre a linha da cirurgia de quadril. Fonte: própria, 2015

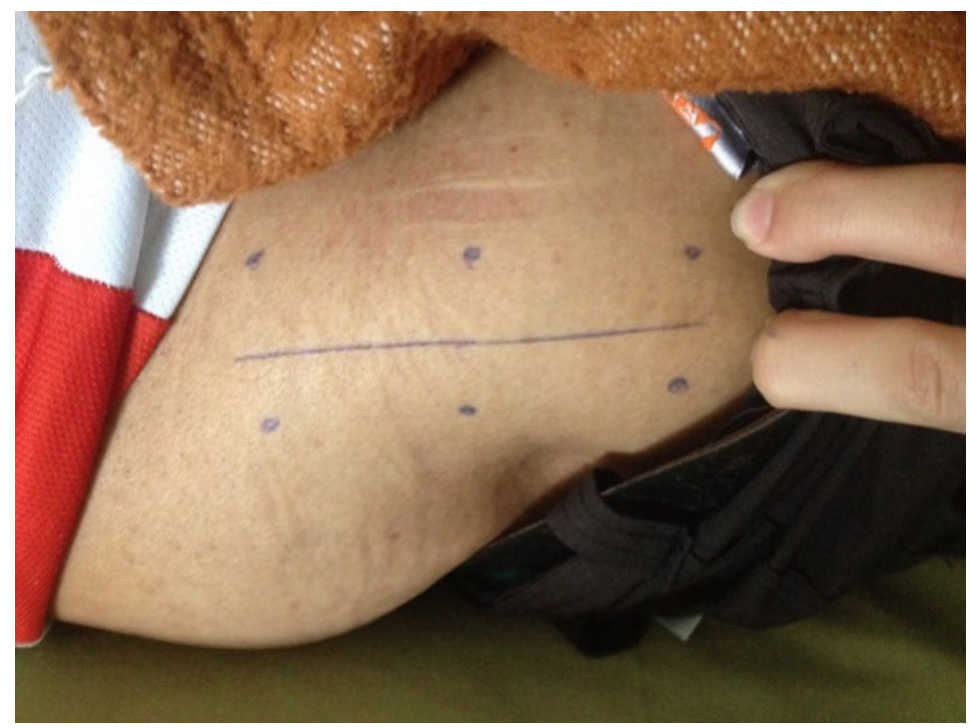

Foi feita uma familiarização com o equipamento na mão ou no antebraço do participante antes do início de cada coleta. Assim que a dor era percebida pelo participante, o ponteiro do algômetro era paralisado. Foram realizadas 3 tentativas com intervalo de 60 segundos. A média dessas tentativas foi computada para cada região ${ }^{19}$. Por fim, perguntou-se ao idoso: “O senhor (a) sente dor em algum lugar do corpo?".

Para análise descritiva dos dados, usou-se o programa Statistical Package for the Social Science (SPSS), versão 20.0, e foi aplicado o coeficiente de correlação intraclasse (CCI) 1,1(20) para coletar dados contínuos para amostras relacionadas, tanto para a análise inter quanto para a intraexaminadora. Considerou-se correlação fraca quando o valor achado era menor que 0,4; correlação satisfatória com valor maior ou igual a 0,4 e menor que 0,75. Quando o valor encontrado foi maior ou igual a 0,75 , a correlação foi considerada excelente ${ }^{21}$.

\section{Resultados}

Os dados obtidos foram divididos em quatro tabelas: Na tabela 1, estão os dados sociodemográficos dos idosos; na tabela 2, está a algometria de quadril; na tabela 3, está a algometria intraexaminador e, na tabela 4, estão os interexaminadores.

Quando questionados, por meio do Inventário da Dor, se tinham outras dores no corpo, $41 \%{ }^{7}$ disseram sentir dores na região dos membros inferiores, $24 \%{ }^{4}$ relataram dores nos membros superiores e $35 \%^{6}$ disseram não sentir dor alguma. Os idosos foram arguidos sobre se, na última semana, haviam sentido alguma dor: 70\% ${ }^{12}$ responderam não e $30 \% \underline{5}$ disseram sim. 
Sobre o uso de medicação que os idosos fizeram para dor, 53\%ํํำ disseram que não usavam nenhum medicamento para dor, $41 \%^{\underline{7}}$ não souberam responder e $6 \%^{1}$ disseram que usavam.

Ao se pesquisar o número de quedas, por meio do Histórico de Quedas, esses idosos apresentavam, pelo menos, uma queda no último mês $70 \%^{12}, 24 \%^{4}$ tiveram duas quedas e apenas $6 \%^{1}$ relataram nenhuma queda no último mês.

Ao se investigar a incidência de depressão entre os idosos, por meio da Escala de Depressão Geriátrica, foi constatado que 9 indivíduos idosos (52,94\%) foram enquadrados como depressivos e 8 idosos (47,06\%) foram enquadrados como normais.

Os idosos deste estudo possuem um IMC (índice de massa corpórea) dentro da média de 27,4. Os demais dados sociodemográficos e de saúde estão na tabela 1:

Tabela 1. Dados sociodemográficos dos idosos do estudo $(n=17)$

\begin{tabular}{|c|c|}
\hline Variáveis & $\begin{array}{l}\text { Valores } \\
\text { (n) (\%) }\end{array}$ \\
\hline Idade (média) & $\pm 73,7$ \\
\hline Idade (70 a 89 anos) & $11(64,7)$ \\
\hline Sexo (feminino) & $10(58,8)$ \\
\hline Uso de 3 a 5 medicações & $12(70,6)$ \\
\hline Não usa dispositivo de auxílio de marcha & $12(70,6)$ \\
\hline Percepção subjetiva de visão boa a regular & $15(88,3)$ \\
\hline Percepção subjetiva de audição boa a regular & $11(64,7)$ \\
\hline IMC & \\
\hline Obesidade & $8(47,0)$ \\
\hline Peso normal & $5(29,4)$ \\
\hline Condições de Saúde & \\
\hline Incontinência Urinária (presente) & $9(52,9)$ \\
\hline Problemas para dormir (presente) & $4(35,3)$ \\
\hline ICF & \\
\hline Osteoartrite e Osteoartrose (presente) & $7(41,2)$ \\
\hline Depressão (presente) & $6(35,3)$ \\
\hline MEEM & \\
\hline Pontuaram >22 a 25 ( 1 a 4 anos de escolaridade) & $10(58,8)$ \\
\hline Pontuaram $<20$ (analfabetos) & $4(23,5)$ \\
\hline GDS & \\
\hline Apresentam sintomas de depressão & $9(52,9)$ \\
\hline SPPB & \\
\hline Pontuaram de 5 a 7 baixa capacidade física & $10(58,7)$ \\
\hline TIME UP GO TEST & \\
\hline Levaram até 2 minutos para realizer o teste & $8(47,0)$ \\
\hline DOR & \\
\hline Sente dor & $10(58,8)$ \\
\hline Dor no Ombro & $4(23,5)$ \\
\hline Dor no Joelho & $6(35,3)$ \\
\hline Sentiu dor na última semana & $5(29,4)$ \\
\hline Não está sob efeito de medicamento & $12(70,6)$ \\
\hline Histórico de Quedas & \\
\hline Caiu nos últimos 12 meses & $8(47,1)$ \\
\hline Apresentou dor por causa da queda & $3(17,6)$ \\
\hline
\end{tabular}

*IMC (Índice de Massa Corporal); *ICF (Índice de Comorbidades Funcionais); *MEEM (Mini Exame do Estado Mental); *GDS (Escala de Depressão Geriátrica); *SPPB (Short Physical Performance Battery); 
Tabela 2. Média da algometria realizada intra e interavaliador. *Avaliação intra-avaliador primeira medida; **Avaliação intra-avaliador após 1 semana; ***Avaliação interavaliador no mesmo dia que o avaliador $\mathrm{A}$

\begin{tabular}{llll}
\hline Idoso & Algometria A & Algometria A** & Algometria B*** \\
\hline $\mathbf{1}$ & 26,05 & 28,76 & 16,1 \\
$\mathbf{2}$ & 42,72 & 56,66 & 43,93 \\
$\mathbf{3}$ & 27,36 & 33,9 & 28,76 \\
$\mathbf{4}$ & 23,82 & 50,25 & 25,24 \\
$\mathbf{5}$ & 29,28 & 13,16 & 25,68 \\
$\mathbf{6}$ & 20,01 & 25,95 & 17,53 \\
$\mathbf{7}$ & 6,15 & 6,38 & 3,51 \\
$\mathbf{8}$ & 7,91 & 13,68 & 5,51 \\
$\mathbf{9}$ & 8,25 & 7,87 & 7,12 \\
$\mathbf{1 0}$ & 4,34 & 7,29 & 3,86 \\
$\mathbf{1 1}$ & 7,36 & 6,26 & 5,44 \\
$\mathbf{1 2}$ & 4,65 & 5,82 & 4,05 \\
$\mathbf{1 3}$ & 6,49 & 8,94 & 7,52 \\
$\mathbf{1 4}$ & 9,46 & 13,27 & 7,57 \\
$\mathbf{1 5}$ & 23,22 & 40,79 & 27,07 \\
$\mathbf{1 6}$ & 38,08 & 47 & 26,79 \\
$\mathbf{1 7}$ & 18,32 & 4,33 & 13,67 \\
\hline
\end{tabular}

Tabela 3. Correlação Intraexaminador

\begin{tabular}{lll}
\hline & Correlação Intraexaminador & ICC 95\% \\
\hline Resultado & 0,877 & 0,956 \\
\hline Nota: Coeficiente de Correlação Intraclasse &
\end{tabular}

Tabela 4. Correlação Interexaminador

\begin{tabular}{lll}
\hline & Correlação Interexaminador & ICC 95\% \\
\hline Resultado & 0,973 & 0,990 \\
\hline Nota: Coeficiente de Correlação Intraclasse &
\end{tabular}

\section{Discussão}

Mensurou-se a sensibilidade do algômetro intra e interexaminador em avaliar e quantificar o limiar de dor em idosos residentes de duas instituições de longa permanência. A escolha legitima-se pelo fato desses serem mais fragilizados e apresentarem maiores morbidades físicas ou mentais ${ }^{22}$, devido ao isolamento social, à inatividade física e a processos psicológicos, logo, deduz-se que, quanto maior o tempo de institucionalização, maior a debilidade do idoso $\underline{23}$.

A dor é uma das principais consequências da queda em idosos ${ }^{24}$, sendo que, no Brasil, cerca de $30 \%$ sofrem quedas ao menos uma vez no ano ${ }^{25}$. Distúrbios musculoesqueléticos, como osteoartrose, que resultam em rigidez e dor nas articulações, estão ligadas à instabilidade no equilíbrio $\underline{26}$, podendo levar à queda.

O estudo populacional SABE, sobre dor crônica em idosos de São Paulo, mostraram, pela primeira vez, que a dor é um acometimento importante para os idosos e pode levar a incapacidades importantes na sociedade ${ }^{27}$.

A dor crônica traz impactos negativos sobre a saúde física e mental dos idosos, tais como depressão, incapacidade física, dependência funcional e isolamento social28, sobrecarregando os serviços de saúde ${ }^{13}$. Particularmente, a dor crônica aumenta o risco de quedas recorrentes em idosos ${ }^{29}$. Existem poucos estudos sobre dor crônica em idosos. Isso pode ser porque exista o mito de que idosos são mais sensíveis e queixosos para dor e a mesma passa a ser vista como uma característica normal do envelhecimento e que, muitas vezes, não pode e nem precisam ser tratadas ${ }^{30}$. 
A associação entre dor e prejuízo cognitivo residem no fato de que áreas cerebrais envolvidas nos mecanismos regulatórios de dor são as mesmas envolvidas na tomada de decisão e na atenção ${ }^{31}$. Além disso, a maioria dos pacientes com dor crônica também sofre de depressão, ansiedade, estresse ou a combinação desses fatores, afetando, diretamente, o funcionamento cognitivo por meio da apatia, de efeitos da medicação, da fadiga e de distúrbios do sono ${ }^{32}$.

A dor crônica em idosos é um problema de saúde frequente encontrado pelos profissionais da saúde que lidam com idosos, principalmente, entre idosos acima de 65 anos. A dor de característica crônica associa-se à incapacidade funcional; à redução da mobilidade e de atividades do dia a dia; ao risco de quedas; à depressão e à ansiedade; a problemas com o sono e até ao isolamento social ${ }^{33}$.

A dor precisa ser avaliada para além de um evento sensorial. Ela possui características afetivas (que são as respostas emocionais frente ao quadro crônico), cognitivas (que envolvem as atitudes e crenças sobre a dor), comportamentais (que envolvem as atitudes do idoso bem como de seus familiares e cuidadores frente à dor) e os componentes sensoriais (que têm a ver com a qualidade, localização e modo dessa dor) ${ }^{28}$.

Um estudo ${ }^{33}$ sobre a dor crônica de idosos observou que a região dorsal foi o local predominante (48\%), seguida dos membros inferiores (24\%), cabeça (8\%) e membros superiores (4\%). Os idosos deste estudo também apresentaram dores em membros inferiores.

O principal tratamento para a dor crônica tem sido a medicação, porém, para o idoso, essa não tem sido a melhor opção devido ao alto custo, aos efeitos colaterais e à baixa eficiência de melhora, o que fomenta a procura por fisioterapia e psicoterapia $34-35$, embora a maioria dos idosos queixosos de dor, neste estudo, não usem nenhuma medicação para dor. Porém, quando questionados sobre uso de outras medicações, relataram usar anti-hipertensivo, antidepressivo, ansiolítico, entre outros, o que pode justificar a alta prevalência de quedas nesses idosos. Sabe-se que essas drogas diminuem o alerta, a função psicomotora, podendo causar fraqueza muscular, tontura, hipotensão postural, principalmente, se administradas em doses inapropriadas ${ }^{36-37}$.
Eventualmente, os idosos avaliados apresentaram, pelo menos, uma queda no último ano. Esses idosos têm maior probabilidade de sofrer quedas, quando comparados a idosos não institucionalizados, por possuírem menores níveis de força, equilíbrio, flexibilidade e resistência física ${ }^{38}$.

Os idosos desse estudo apresentaram excesso de peso, fatores importantes para desenvolvimento de dor e aumento do risco de queda. Um estudo ${ }^{39}$, que teve como objetivo identificar o estado nutricional, segundo o índice de massa corporal de idosos institucionalizados, observou que $66,5 \%$ da amostra tinha um estado nutricional inadequado, sendo $45,5 \%$ de baixo peso, $33,5 \%$ de peso normal, $7,8 \%$ de pré-obesidade e $13,2 \%$ de obesidade ${ }^{39}$. Observamos que nossos dados correspondem à literatura, uma vez que a média do IMC $=27,4$ corresponde ao excesso de peso ${ }^{40}$.

Os idosos apresentaram sintomas depressivos que podem piorar a sensação de dor, a prevalência de sintomas de depressão detectada foi menor que a relatada entre os idosos da cidade de Juazeiro do Norte, Ceará, onde foi verificada uma prevalência de depressão de $65 \%$, porém, maior que nos idosos do distrito de Bragança que apresentaram uma incidência de $46,7 \%$ \%

O resultado obtido para intraexaminadores, neste estudo, foi igual a 0,87 , indicando uma alta confiabilidade do instrumento em avaliar o limiar de dor em idosos quando realizada pelo mesmo examinador no intervalo de uma semana entre as medidas.

Quanto à confiabilidade interexamiandores, o resultado obtido foi igual a 0,97 , o que indica uma confiabilidade muito alta do instrumento quando feito por avaliadores distintos.

Ter um aparelho que possa mensurar e quantificar o limiar da dor em idosos retira o caráter subjetivo quando se avalia dor em idosos apenas por escalas e questionários. Para profissionais da saúde, a mensuração do limiar da dor pode indicar o bem-estar do paciente, servindo como parâmetro para sua evolução e auxiliando na escolha da medicação e diagnóstico ${ }^{43}$. 


\section{Conclusão}

O algômetro digital da marca Instrutherm DD-200® mostrou-se sensível em mensurar o limiar de dor por pressão na região do quadril em idosos residentes em uma instituição de longa permanência. Sabe-se quanto menor for em newtons (mesma direção, sentido e força) a sensibilidade do idoso ao algômetro maior será a sua dor no local testado. No entanto, estudos com uma população maior precisam ser realizados.

\section{Agradecimentos}

À professora Beatriz Fraga pelas correções ortográficas e gramaticais do texto, sua vivência com o envelhecimento fizeram essas correções especiais.

\section{Contribuições dos autores}

Prestes YA participou da concepção, delineamento e redação do artigo científico. Souza MS participou do delineamento e análise dos dados. Pereira MS e Ervati R participaram da concepção, delineamento, aquisição e análise estatística dos dados da pesquisa, interpretação dos resultados, redação do artigo científico. Campos HLM participou da concepção da pesquisa, orientou no delineamento e análise estatística dos dados da pesquisa, orientou na interpretação dos resultados e supervisionou a redação do artigo científico.

\section{Conflitos de interesses}

Nenhum conflito financeiro, legal ou político envolvendo terceiros (governo, empresas e fundações privadas, etc.) foi declarado para nenhum aspecto do trabalho submetido (incluindo, mas não se limitando a subvenções e financiamentos, participação em conselho consultivo, desenho de estudo, preparação de manuscrito, análise estatística, etc.).

\section{Referências}

1. Instituto Brasileiro de Geografia e Estatística. Contas Nacionais - Conta-Satélite de Saúde 2007- 2009. [Internet]. 2012. Disponível em: https://biblioteca.ibge.gov.br/visualizacao/livros/liv101437.pdf

2. World Health Organization. Envelhecimento e Saúde. [Internet]. 2018. [Acesso em 2019 nov. 08]. Disponível em: http://www.who. int/news-room/fact-sheets/detail/ageing-and-health
3. Instituto Brasileiro de Geografia e Estatística. Tendência do envelhecimento dos brasileiros. [Acesso em 28 dez 2019] Disponível em: https://censo2020.ibge.gov.br/2012-agencia-denoticias/noticias/24036-idosos-indicam-caminhos-para-umamelhor-idade.html

4. Ministério da Saúde. Agência Nacional de Vigilância Sanitária. Resolução n. ${ }^{\circ} 283$ de 26 de setembro de 2005. Aprova o Regulamento Teórico que define normas de funcionamento para Instituições de Longa Permanência para Idosos. [Internet]. 2005. Diário Oficial da União. Disponível em: https://sbgg.org.br/wpcontent/uploads/2014/10/rdc-283-2005.pdf

5. Gold DT, Roberto KA. Correlates and consequences of chronic pain in older adults. Geriatr Nurs. 2000;21(5):270-3. doi: 10.1067/ mgn.2000.110838

6. Beissner K, Henderson Jr CR, Papaleontioul M, Olkhovskaya Y, Wigglesworth J, Reld MC. Physical Therapists use of cognitivebehavioral therapyfor older adults with chronic pain: a nationwide survey. Phys Ther. 2009;89(5):456-69. doi: 10.2522/ptj.20080163

7. Harris T, Cook DG, Victor C, DeWilde S, Beighton C. Onset and persistence of depression in older people-results froma 2-year community follow-up study. Age Ageing. 2006;35(1):25-32. doi: 10.1093/ageing/afi216

8. Stel VS, Smit JH, Pluijm SMF, Lips P. Consequences of falling in older men and women and risk factors for health service use and functional decline. Age Ageing. 2004;33(1):58-65. doi: 10.1093/ ageing/afh028

9. Silva A, Almeida GJM, Cassilhas RC, Cohen M, Peccin MS, Tufik $S$ et al. Equilíbrio, coordenação e agilidade de idosos submetidos à prática de exercícios físicos resistidos. Rev Bras Med Esporte. 2008;14(2):88-93. doi: 10.1590/S1517-86922008000200001

10. Blumstein $\mathrm{H}$, Gorevic PD. Rhematologic illnesses: treatment strategies tor older adults. Geriatrics. 2005;60(6):28-35.

11. Souza RC, Pinheiro RS, Coeli CM, Camargo Jr KR, Torres TZG. Aplicação de medidas de ajuste de risco para a mortalidade após fratura proximal de fêmur. Rev Saúde Pública. 2007; 41(4)625-631. doi: 10.1590/S0034-89102007000400017

12. Cruz DT, Ribeiro LC, Vieira MT, Teixeira MTB, Bastos RR, Leite ICG. Prevalência de quedas e fatores associados em idosos. Rev Saúde Pública. 2012;46(1):138-146. doi: 10.1590/S0034$\underline{89102011005000087}$

13. Dellaroza MSG, Furuya RK, Cabrera MAS, Matsuo T, Trelha C, Yamada KN et al. Caracterização da dor crônica e métodos analgésicos utilizados por idosos da comunidade. Rev Assoc Med Bras. 2008;54(1):36-41. doi: 10.1590/S0104-42302008000100018

14. Carvalho EMR, Garcês JR, Menezes RL, Silva ECF. O olhar e o sentir do idoso no pós-queda. Rev Bras Geriatr Gerontol. 2010;13(1):7-16. doi: 10.1590/S1809-98232010000100002 
15. Brucki SMD, Nitrini R, Caramelli P, Bertolucci PHF, Okamoto IH. Sugestões para uso de Mini-Exame de Estado Mental no Brasil. Arq Neuropsiquiatr. 2003;61(3-B):777-781. doi: 10.1590/50004$\underline{282 \times 2003000500014}$

16. Apóstolo JLA, Campos EMB, Reis IAC, Henriques SJ, Correia CAV. Capacidade de rastreio da Escala de Depressão Geriátrica com 10 e 5 itens. Rev Enf Ref. 2018;4(16):29-40. doi: 10.12707/ RIV17062

17. Moraes EN, Carmo JA, Moraes FL, Azevedo RS, Machado CJ, Montilla DER. Índice de Vulnerabilidade Clínico Funcional-20 (IVCF20): reconhecimento rápido do idoso frágil. Rev Saúde Pública. 2016;50:81. doi: 10.1590/S1518-8787.2016050006963

18. Silva TO, Freitas RS, Monteiro MR, Borges SM. Avaliação da capacidade física e quedas em idosos ativos e sedentários da comunidade. Rev Bras Clin Med. 2010;8(5):392-8.

19. Martin F, Cherif K, Gentili ME, Enel D, Abe E, Alvarez JC et al. Lack of impact of intravenous lidocaine on analgesia, functional recovery, and nociceptive pain threshold after total hip arthroplasty. Anesthesiology. 2008;109(1):118-123. doi: 10.1097/ ALN.0b013e31817b5a9b

20. Weir JP. Quantifying test-retest reliability using the intraclass correlation coefficient and the SEM. J Strenght Cond Res. 2005;19(1):231-40.

21. Fleiss JL. The design and analysis of clinical experiments. New York: Wiley; 1986.

22. Alexandre NMC, Gallasch CH, Lima MHM, Rodrigues RCM. Confiabilidade no desenvolvimento e avaliação de instrumentos de medida na área da saúde. Rev Eletr Enf. 2013;15(3): 802-9. doi: 10.5216/ree.v15i3.20776

23. Tideiksaar R. As quedas na velhice: prevenção e cuidados. 2.ed. São Paulo: Organização Andrei Editora Ltda; 2003.

24. Cunha UGV, Guimarães RM. Sinais e sintomas do aparelho locomotor. In: Guimarães RM, Cunha UGV. Sinais e sintomas em geriatria. Rio de Janeiro: Revinter; 1989. p. 141-54.

25. Merskey H. Classification of chronic pain: descriptions of chronic pain syndromes and definitions of pain terms prepared by the International Association for the Study of Pain. Pain Suppl. 1986;3:S1-226.

26. Barry LC, Gill TM, Kerns RD, Reid MC. Identification of painreduction strategies used by community-dwelling older persons. J Gerontol A Biol Sci Med Sci. 2005;60(12): 1569-75. doi: 10.1093/ gerona/60.12.1569

27. Ramos LR, Rosa TEC, Oliveira ZM, Medina MCG, Santos FRG. Perfil do idoso em área metropolitana na região sudeste do Brasil: resultados de inquérito domiciliar. Rev Saúde Pública. 1993;27(2):87-94. doi: 10.1590/50034-89101993000200003
28. Reid MC, Eccleston C, Pillemer K. Management of chronic pain in older adults. BMJ. 2015;350:532. doi: 10.1136/bmj.h532

29. Stubbs B, Binnekade T, Eggermont L, Sepehry AA, Patchay $S$, Schofield P. Pain and the risk for falls in community-dwelling older adults: systematic review and meta-analysis. Arch Phys Med Rehabil. 2014;95(1):175-187. doi: 10.1016/j.apmr.2013.08.241

30. Santos CC, Pereira LSM, Resende MA, Magno F, Aguiar V. Aplicação da versão brasileira do questionário de dor Mcgill em idosos com dor crônica Applicability of the Brazilian version of the McGill pain questio-nnaire in elderly patients with chronic pain. Acta Fisiatr. 2006;13(2):75-82.

31. Gomes GAO, Cintra MJDD, Neri ALG, Sousa MLR. Comparação entre idosos que sofreram quedas segundo desempenho físico e número de ocorrências. Rev Bras Fisioter. 2009;13(5):430-7. doi: 10.1590/S1413-35552009005000059

32. Nes LS, Roach AR, Segerstrom SC. Executive functions, self-regulation, and chronic pain: a review. Ann Behav Med. 2009;37(2):173-83. doi: 10.1007/s12160-009-9096-5

33. Pinto MVM. Enfoque da Fisioterapia Preventiva: Perfil dos idosos, asilamento e importância do apoio familiar, em CaratingaMG. Fisioter Bras. 2003;4(5):353-9. doi: 10.33233/fb.v4i5.3050

34. Lacerda PF, Godoy LF, Cobianchi MG, Bachion MM. Estudo da ocorrência de "dor crônica" em idosos de uma comunidade atendida pelo programa saúde da família em goiânia. Revista Eletrônica de Enfermagem. 2006;7(1):29-40. doi: 10.5216/ree. v7i1.863

35. Bertolucci PHF, Brucki SMD, Campacci SR, Juliano Y. O MiniExame do Estado Mental em uma população geral: impacto da escolaridade. Arq Neuro-Psiquiatr. 1994; 52(1):01-07. doi: 10.1590/S0004-282X1994000100001

36. Pereira LSM, Britto RR, Valadares NC. Programa de Melhoria da Qualidade de Vida dos Idosos Institucionalizados. In: Anais do $7^{\circ}$ Encontro de Extensão da Universidade Federal de Minas Gerais; 2004.

37. Gawryszewski VP. A importância das quedas no mesmo nível entre idosos no Estado de São Paulo. Rev Assoc Med Bras. 2010;56(2):162-7. doi: 10.1590/S0104-42302010000200013

38. Tinetti ME, Baker DI, McAvay G, Claus EB, Garrett P, Gottschalk $M$ et al. A multifactorial intervention to reduce the risk of falling among elderly people living in the community. N Engl J Med. 1994;331(13):821-7. doi: 10.1056/NEJM199409293311301

39. Rekeneire N, Visser M, Peila R, Nevitt MC, Cauley JA, Tylavsky FA et al. Is a fall just a fall: correlates of falling in healthy older persons. The health, aging and body composition study. J Am Geriatr Soc. 2003;51(6):841-6. doi: 10.1046/j.13652389.2003.51267.x 
40. Rauen MS, Moreira EAM, Calvo MCM, Lobo AS. Avaliação do estado nutricional de idosos institucionalizados. Rev Nutr. 2008; 21(3):303-310. doi: 10.1590/S1415-52732008000300005

41. Wold Health Organization. Physical status: the use and interpretation of anthropometry. [Internet]. 1995. Disponível em https://bit.ly/2EejEpM
42. Nascimento DC, Brito MAC, Santos AD. Depressão em idosos residentes em uma instituição asilar na cidade de Juazeiro do Norte, Ceará, Brasil. J Manag Prim Health Care. 2013;4(3):146-150. doi: $10.14295 /$ jmphc.v4i3.182

43. Vaz SFA, Gaspar NMS. Depressão em idosos institucionalizados no distrito de Bragança. Rev Enf Ref. 2011;(4):49-58. doi: 10.12707/RIII1124 